PAPER REFERENCE: H-III.3

\title{
INVESTIGATION OF DEFECTS AND SURFACE POLARITY IN GaN USING HOT WET ETCHING TOGETHER WITH MICROSCOPY AND DIFFRACTION TECHNIQUES
}

\author{
P. Visconti ${ }^{1,2,3}$, D. Huang ${ }^{1}$, M. A. Reshchikov ${ }^{1}$, F.Yun $^{1}$, R. Cingolani ${ }^{2}$, D. \\ J. Smith ${ }^{4}$, J. Jasinski ${ }^{5}$, W. Swider ${ }^{5}$, Z. Liliental-Weber ${ }^{5}$ and H. Morkoç ${ }^{1}$ \\ ${ }^{1}$ VCU, Dept. of Electrical Engineering, Richmond, 601 W. Main Street, \\ 23284, VA, USA \\ ${ }^{2}$ National Nanotechnology Laboratory of INFM - NNL and Dept. of Innovation \\ Engineering, Univ. of Lecce, Via per Arnesano, 73100, Lecce ITALY \\ ${ }^{3}$ Istituto per lo Studio di Nuovi Materiali per l'Elettronica - CNR, Via per \\ Arnesano, 73100, Lecce, ITALY \\ ${ }^{4}$ Dept. of Physics and Astronomy. Center for Solid State Science, Arizona \\ State Univ., Tempe, AZ \\ ${ }^{5}$ Lawrence Berkeley National Laboratory, Berkeley, CA
}

\begin{abstract}
The availability of reliable and quick methods to determine defect density and polarity in GaN films is of great interest. We have used photo-electrochemical (PEC) and hot wet etching using $\mathrm{H}_{3} \mathrm{PO}_{4}$ and molten $\mathrm{KOH}$ to estimate the defect density in $\mathrm{GaN}$ films grown by hydride vapor phase epitaxy (HVPE) and molecular beam epitaxy (MBE). Free-standing whiskers and hexagonal etch pits are formed by PEC and wet etching respectively. Using Atomic Force Microscopy (AFM), we found the whisker density to be similar to etch pit densities for samples etched under precise conditions. Additionally Transmission Electron Microscopy (TEM) observations confirmed dislocation
\end{abstract}


densities obtained by etching which increased our confidence in the consistency of methods used. Hot wet etching was used also to investigate the polarity of $\mathrm{GaN}$ films together with Convergent Beam Electron Diffraction (CBED) and AFM imaging. We found that hot $\mathrm{H}_{3} \mathrm{PO}_{4}$ etches N-polarity GaN films very quickly resulting in the complete removal or drastic change of surface morphology as revealed by AFM or optical microscopy. On the contrary, the acid attacks only defect sites in Gapolarity films producing nanometer-scale pits but leaving the defect-free GaN intact and the morphology unchanged. Additionally, the polarity assignments were related to the as-grown morphology and to the growth conditions of the buffer layer and the subsequent GaN layer.

\section{Introduction}

Successful fabrication of GaN-based devices depends on the ability to grow epitaxial films on sapphire or silicon carbide with low defect density. The poor match in both lattice parameter and thermal expansion coefficient results in a high density of threading dislocations (TD) $\left(10^{8}-10^{10} \mathrm{~cm}^{-}\right.$ $\left.{ }^{2}\right)[1,2,3,4,5]$ that have been demonstrated to affect both electrical and optical properties of the material [6,7]. Mostly, the characterization of dislocations is carried out using TEM, a process that requires extensive sample preparation. Wurtzite $\mathrm{GaN}$ is a polar material that has two different planes along its c axis. The (0001) plane is the Ga-terminated face while the (000-1) plane is the Nface. It is known that the surface and bulk properties of epitaxial GaN layers depend greatly on the polarity $[8,9]$. CBED, X-ray photoelectron spectroscopy, coaxial impact collision ion scattering spectroscopy [10], X-ray standing wave method [11], in-situ reflection high-energy electron diffraction (RHEED) [12,13] and wet etching [14,15,16,17] have been used to determine the polarity. Particularly, $\mathrm{NaOH}$ and $\mathrm{KOH}$ based aqueous solutions at different temperatures have been demonstrated to attack N-polar surface whereas the morphology of Ga-polar surface remains unchanged. The availability of reliable and quick methods to determine the defect density and the 
surface polarity of GaN films is of great importance in order to understand the correlation between polarity and growth conditions and consequently to achieve high-quality GaN layers.

Wet chemical etching is a convenient technique to determine the density of defects propagating to the surface. Hot $\mathrm{H}_{3} \mathrm{PO}_{4}$, mixed $\mathrm{H}_{3} \mathrm{PO}_{4} / \mathrm{H}_{2} \mathrm{SO}_{4}$ solution and molten $\mathrm{KOH}$ have been found to etch pits at the surface defect sites $[18,19,20]$. However the origin of etch pits is still controversial and the obtained etch pit densities (EPD) $\left(4 \times 10^{5}-1 \times 10^{8} \mathrm{~cm}^{-2}\right)$ is lower than the dislocation density (DD) $\left(10^{8}-10^{10} \mathrm{~cm}^{-2}\right)$ found by TEM. Recently, Youtsey et al [21,22] demonstrated PEC etching for DD estimation in n-type GaN films. They reported nanometer-scale whiskers obtained by selectively etching $\mathrm{GaN}$ between dislocation sites and, with TEM analysis, demonstrated the whisker density to be very close to the effective DD. We have investigated defects in GaN films by PEC method and wet etching using both $\mathrm{H}_{3} \mathrm{PO}_{4}$ and molten $\mathrm{KOH}$. Our purpose is to determine whether, and under what conditions these techniques are consistent in order to get to a better estimation of the defect density. We found the whisker density to be similar to the EPDs for samples etched under precise conditions. Additionally TEM observations confirmed DDs obtained by etching which increased our confidence in the consistency of methods used [21]. Because the etching procedures are simpler and less time consuming, they can be an excellent precursor to TEM analysis for determination of the DD.

Additionally, we have demonstrated that hot $\mathrm{H}_{3} \mathrm{PO}_{4}$ can be used also to determine the polarity of GaN films. In fact hot $\mathrm{H}_{3} \mathrm{PO}_{4}$ etches $\mathrm{N}$-polarity $\mathrm{GaN}$ films very quickly resulting in the complete removal or a drastic change of the surface morphology. On the contrary, the acid attacks only the defect sites in Ga-polarity films leaving the defect-free GaN intact and the morphology unchanged. The polarity assignments, confirmed by CBED experiments, were related to the asgrown morphology and to the growth conditions. We found that by growing GaN with MBE on AlN or GaN buffer layer, it is possible to get Ga or N-polarity depending on the growth temperature 
and growth rate of the buffer layers. GaN films grown on high temperature (HT) AlN or GaN buffer layers show Ga- and N-polarity, respectively. The films grown using low temperature (LT) buffer layer could have either Ga- or N-polarity, depending on the growth rate of the buffer layer. An AlN buffer layer grown at $\sim 60 \mathrm{~nm} /$ hour led to a Ga-polar film. When the growth rate was reduced to $\sim 20$ $\mathrm{nm} /$ hour, a N-polar film could result. Similarly, a GaN buffer layer grown at $\sim 220 \mathrm{~nm} /$ hour led to a $\mathrm{N}$-polar film. When the growth rate was raised to $\sim 600 \mathrm{~nm} /$ hour, a Ga-polar film could be obtained.

\section{Experimental details}

Two different sets of GaN samples were used for the experiments. The first set was Sidoped $\left(\mathrm{n} \sim 2 \times 10^{18} \mathrm{~cm}^{-3}\right) \approx 9 \mu \mathrm{m}$ thick Ga-polar GaN layers grown by HVPE on sapphire. The second set consisted of unintentionally n-doped GaN layers grown by MBE on sapphire. Nitridation was performed at high $\left(890-985^{\circ} \mathrm{C}\right)$ and low $\left(\approx 500{ }^{\circ} \mathrm{C}\right)$ temperatures by radio frequency $(\mathrm{RF}) \mathrm{N}$ plasma, which have no apparent effect on the results. Some samples utilized GaN buffer layers grown at $500{ }^{\circ} \mathrm{C}$, and $800{ }^{\circ} \mathrm{C}$. Others utilized AlN buffer layers grown near $500{ }^{\circ} \mathrm{C}$ and $890-930{ }^{\circ} \mathrm{C}$. Following the buffer layers, $\approx 1 \mu \mathrm{m}$ thick GaN layers were grown at a temperature between 720 and $850{ }^{\circ} \mathrm{C}$ with growth rates in the range $0.3-1 \mu \mathrm{m} / \mathrm{h}$ under $\mathrm{N}$-limited (Ga-rich) conditions.

PEC etching was carried out in a standard electrochemical cell at room temperature using an unstirred 0.02 M KOH solution and a He-Cd laser for UV illumination. A Ti mask, resistant to the etchant, was patterned around the periphery of the sample with a lift-off process. The Ti contact served to assist the photocurrent conduction. No additional bias was applied between the sample and the cathode. The morphology of GaN samples, etched by PEC and hot wet etching, was investigated using AFM and scanning electron microscopy (SEM). Additionally, some samples were observed by TEM to estimate the DD. The polarity of MBE-grown GaN films was determined by hot $\mathrm{H}_{3} \mathrm{PO}_{4}$ etching, CBED experiments and AFM investigation of as-grown surface morphology. 


\section{Results and Discussion}

\subsection{Defect density determined by PEC and hot wet etching in HVPE-grown GaN films.}

Slightly carrier-limited conditions with moderate illumination intensity were used to etch crystalline GaN material selectively, leaving vertical wires on the surface. The AFM image of figure 1a reveals the PEC etched surface morphology of the HVPE-grown sample. We estimated the height of whiskers to be $\approx 700 \mathrm{~nm}$ and the lateral size $\approx 100 \mathrm{~nm}$. The density is about $1-2 \times 10^{9} \mathrm{~cm}^{-}$

${ }^{2}$. The etched surface morphology was also investigated by SEM (Fig. 1b) The calculated density of features (white dots) is $\approx 2 \times 10^{9} \mathrm{~cm}^{-2}$, the same value obtained from AFM.
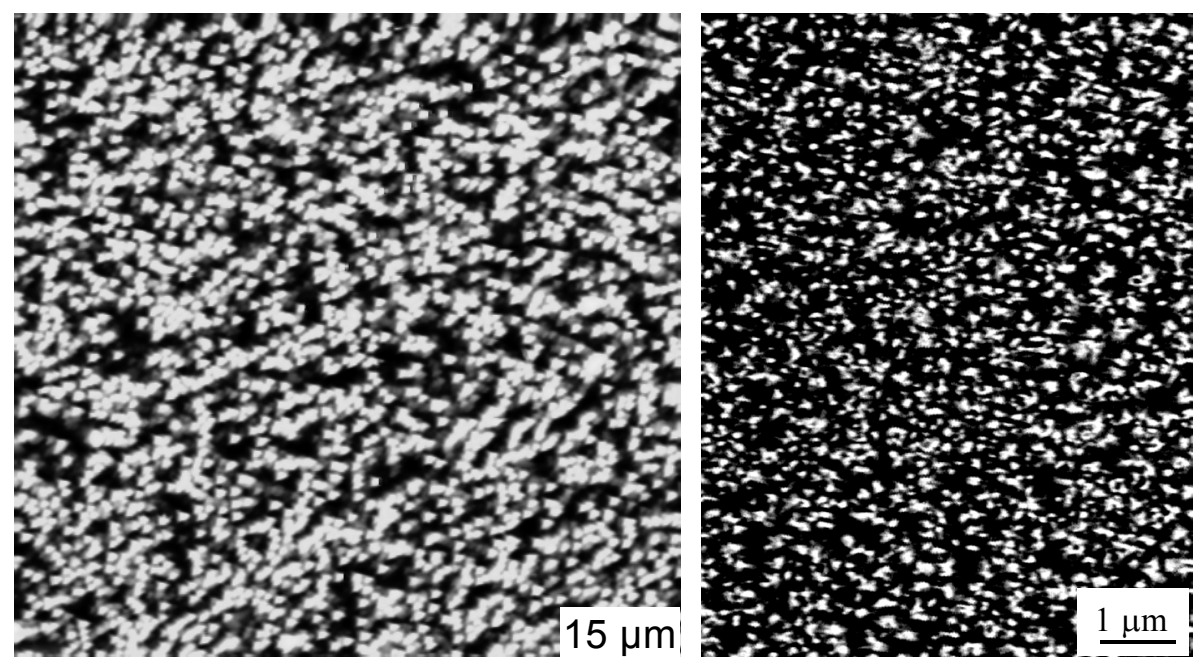

FIG. 1 (a) AFM image of HVPE-grown GaN sample etched by PEC process. Whisker-like features are formed by etching with a density of $\approx 2 \times 10^{9} \mathrm{~cm}^{-2}$. The vertical scale ranges from 0 to $1200 \mathrm{~nm}$. (b) Plan-view SEM image of the PEC etched sample. The density of whiskers (white dots) is $\sim 1-2 \times 10^{9} \mathrm{~cm}^{-2}$ the same value obtained from AFM.

In order to clarify the relation between EPD and DD and look for any consistency among the various etches, we used $\mathrm{H}_{3} \mathrm{PO}_{4}$ and molten $\mathrm{KOH}$ as defect etchants in $\mathrm{GaN}$. The AFM image of the HVPE-grown sample etched by molten $\mathrm{KOH}$ for 2 minutes at $210{ }^{\circ} \mathrm{C}$ is shown in fig. $2 \mathrm{a}$. The pits, with density of $\approx 1 \times 10^{9} \mathrm{~cm}^{-2}$, are of hexagonal shape and their size ranges from 40 to $100 \mathrm{~nm}$ in diameter and from 10 to $30 \mathrm{~nm}$ in depth. Figure $2 \mathrm{~b}$ shows the surface morphology after etching in 
$\mathrm{H}_{3} \mathrm{PO}_{4}$ for 6 minutes at $160{ }^{\circ} \mathrm{C}$. The EPD is the same found for the $\mathrm{KOH}$ etched sample and close to the density of features formed by PEC etching. During the wet etching, a careful balance must be struck to ensure that every defect is delineated, but not over-etched to cause merging which would lead to an underestimation of the defect density. We show in fig.2c an AFM scan of the same sample etched for $10 \mathrm{~min}$ at $200{ }^{\circ} \mathrm{C}$ in $\mathrm{H}_{3} \mathrm{PO}_{4}$. We estimated the $\mathrm{EPD} \approx 1 \times 10^{8} \mathrm{~cm}^{-2}$, an order of magnitude less than the correct value obtained earlier due to over-etching.

We characterized the HVPE-grown sample using TEM in order to get the effective DD and compare this value with the defect densities found by defect revealing wet etches. TD, primarily of edge or mixed character, were observed starting from the buffer/GaN interface and often stopping within the $9 \mu \mathrm{m}$-thick layer. Hexagonal nanopipes are also seen with sizes in the range 4-10 $\mathrm{nm}$ and density $\approx 5-10 \times 10^{7} \mathrm{~cm}^{-2}$. In all the defect density, close to the top surface, was estimated $\approx 0.5$ $2 \times 10^{9} \mathrm{~cm}^{-2}$, similar to the values obtained by defect revealing etches.
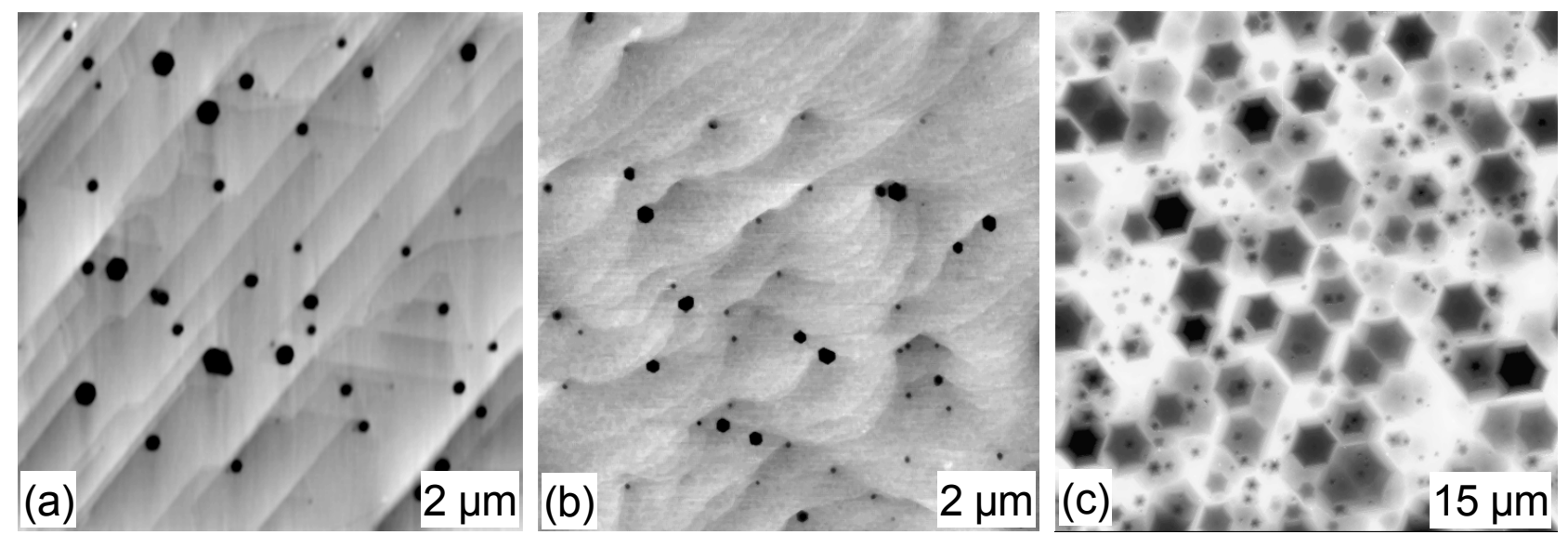

FIG. 2 AFM images of the HVPE-grown GaN surface morphologies produced by wet etching. (a) Surface morphology after etching in molten $\mathrm{KOH}$ for $2 \mathrm{~min}$. at $210{ }^{\circ} \mathrm{C}$. Pits at the defect sites are formed with a density of $1 \times 10^{9} \mathrm{~cm}^{-2}$. (b) Surface morphology after etching in $\mathrm{H}_{3} \mathrm{PO}_{4}$ for $6 \mathrm{~min}$. at $160{ }^{\circ} \mathrm{C}$. The EPD is the same found for the $\mathrm{KOH}$ etched sample. The vertical scale ranges from 0 to $10 \mathrm{~nm}$. (c) Surface morphology after etching in $\mathrm{H}_{3} \mathrm{PO}_{4}$ for $10 \mathrm{~min}$. at $200{ }^{\circ} \mathrm{C}(\mathrm{EPD}$ $\approx 1 \times 10^{8} \mathrm{~cm}^{-2}$ ). The vertical scale ranges from 0 to $450 \mathrm{~nm}$. 


\subsection{Investigation of polarity in MBE-grown GaN films.}

For GaN- based device applications, the understanding and the control of polarity in the epitaxial growth are essential. For MBE growth the published results show that AlN buffer layers commonly lead to Ga-polar films while GaN buffer layers lead to N-polar films. We have studied the dependency of GaN polarity on growth parameters of buffer layer by MBE. We show that both AlN and GaN buffer layers can lead to Ga- and N-polar films depending on the growth temperature and growth rate of buffer layers. The polarity was mainly determined by $\mathrm{H}_{3} \mathrm{PO}_{4}$ wet etching and AFM investigations of both as-grown and etched surface morphology. As reported in the literature, the surface of an as-grown Ga-polar film is either very flat or shows stepped terraces, often with pits. The surface of an as-grown N-polar film by MBE shows tall columns or terraces separated by deep troughs, without pits on the surface. Similarly to $\mathrm{NaOH}$ and $\mathrm{KOH}$-based etchings, we have found that hot $\left(160{ }^{\circ} \mathrm{C}\right) \mathrm{H}_{3} \mathrm{PO}_{4}$ etches $\mathrm{N}$-polar GaN films very quickly resulting in either the complete removal or a drastic change in the surface morphology as revealed by AFM or even by optical microscopy. The etching rate is from 0.3 to $0.7 \mu \mathrm{m} /$ minute. On the contrary, the acid attacks only defect sites in Ga-polarity films leaving the defect-free $\mathrm{GaN}$ intact and the morphology unchanged. The polarity assignments were also supported by in-situ RHEED pattern. A Ga-face usually shows $2 \times 2$ RHEED pattern upon cool-down at temperatures between $280-650^{\circ} \mathrm{C}$ after the entire structure was grown. The RHEED pattern of an N-face upon cooling shows, however, only the bulk 1x1 structure. In addition, CBED experiments confirmed the polarity assignments.

We first examine the polarity of samples grown by MBE on GaN and AlN buffer layers at high temperature (HT). Layers on $\mathrm{HT}\left(>770{ }^{\circ} \mathrm{C}\right) \mathrm{GaN}$ buffer layers invariably turned out to be Npolarity regardless whether a static or graded substrate temperature was employed during the buffer growth. The surface morphology, the wet etching experiments and the CBED analysis are consistent with the n-polarity assignment. The RHEED pattern upon cooling indicates only the bulk 
1x1 structure. Conversely, GaN films grown on $\mathrm{HT}\left(\sim 900^{\circ} \mathrm{C}\right) \mathrm{AlN}$ buffer layers with thicknesses in the range of 8-35 $\mathrm{nm}$ and growth rates of $40-60 \mathrm{~nm} /$ hour led to Ga-polarity. Also in this case, the surface morphology, wet etching experiments, and the CBED study are in agreement with the Gapolarity assignment. The RHEED patterns of $2 \times 2$ reconstruction are observable.

The GaN films grown on low temperature (LT) $\left(\sim 500{ }^{\circ} \mathrm{C}\right)$ buffer layers (either GaN or AlN) were found to have either Ga- or N-polarity. A $60-150 \mathrm{~nm}$ thick GaN buffer layer at a growth rate of about $600 \mathrm{~nm} /$ hour can lead to Ga-polarity as confirmed by the surface morphology and the $2 \times 2$ RHEED pattern. However, when the thickness of buffer layer was reduced to 30-40 nm, keeping the same growth rate, the layers turned out to be of mixed polarity with a faint $2 \times 2$ reconstruction observed upon cool-down. When about $110-220 \mathrm{~nm}$ thick buffer layers were grown at $500{ }^{\circ} \mathrm{C}$ with a lower growth rate $(220 \mathrm{~nm} /$ hour$)$, the resultant layers were of N-polarity with consistent surface morphology revealed by AFM and 1x1 reconstruction being only observed. For GaN films grown on LT $\left(\sim 500^{\circ} \mathrm{C}\right)$ AlN buffer layer, their polarities also depend on the growth rate. When $20 \mathrm{~nm}$ buffer layers grown at rate of $60 \mathrm{~nm} /$ hour were employed, Ga-polar films resulted. However, when 2.5-22 nm thick buffer layers grown at lower rate of 15-30 nm/hour, N-polar films can be obtained.

The typical surface morphologies of as-grown Ga- and N-polar films are presented in fig. 3. A high temperature (HT) AlN buffer layer (Fig. 3a) leads to Ga-polar GaN film with a smooth, but pitted surface morphology. In fig. 3.b is shown the morphology of an N-polar films obtained using a HT GaN buffer layers. The rough morphology present 50-100nm high non-coalesced columns.

X-ray diffraction (XRD) measurements were carried out by a Philips X'Pert MRD system equipped with a four-crystal Ge (220) monochromator. On the average, the symmetric [002] peaks for the Ga-polarity films are sharper than those for the N-polarity films. The difference is much smaller for the [104] asymmetric peaks. In addition, the AlN buffer layers often lead to films with sharper XRD peaks. 

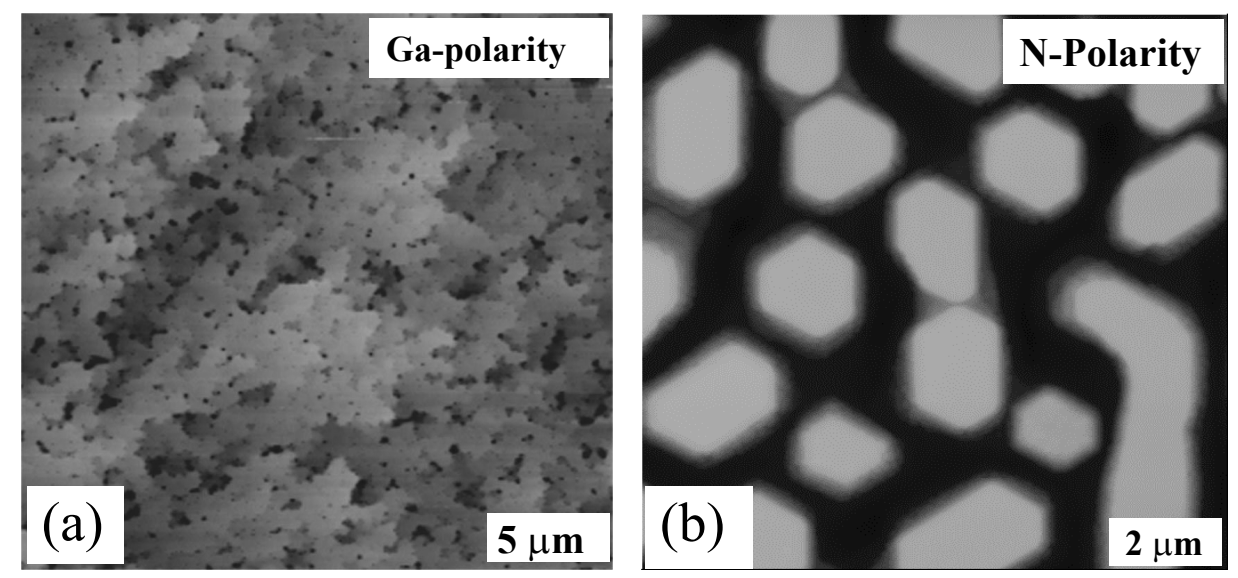

FIG. 3 AFM images of Ga-polar film (fig. a) (rms roughness $\approx 1.3 \mathrm{~nm}$ ) obtained using HT AlN buffer layer and of N-polar film obtained using HT GaN buffer layer (fig. b) (roughness $\approx 20 \mathrm{~nm}$ )

\section{Conclusions}

We have used PEC and hot wet etching to estimate the defect density in GaN films. Freestanding whiskers and hexagonal etch pits are formed by PEC and wet etching. Using AFM, we found the whisker density to be similar to EPDs. TEM study confirmed DD obtained by etching which increased our confidence in the consistency of methods used. Hot wet etching was used also to investigate polarity of $\mathrm{GaN}$ films. $\mathrm{Hot}_{3} \mathrm{PO}_{4}$ etches $\mathrm{N}$-polar $\mathrm{GaN}$ films very quickly resulting in the complete removal or drastic change of surface morphology. On the contrary, the acid attacks only defect sites in Ga-polar films producing nanometer-scale pits but leaving the defect-free $\mathrm{GaN}$ intact and the morphology unchanged. The polarity assignments have been related to the as-grown morphology and to the growth conditions of the buffer layer and the subsequent GaN layer.

\section{Acknowlegements}

The VCU portion of this research was funded by grants from NSF (Drs. L. Hess and G. Pomrenke), AFOSR (Dr. G. L. Witt), and ONR (Drs. C. E. C. Wood and Y. S. Park). The authors would like to thank Prof. R. Feenstra of Carnegie Mellon for useful discussions.

\section{References}

[1] H. Morkoç, S. Strite, G.B. Gao, M.E. Lin, B. Sverdlov, M. Burns, J.Appl.Phys. 76, (1994) 1363.

[2] S. Nakamura, T. Mukai, and M. Senoh, Appl. Phys. Lett. 64, (1994) 1687.

[3] S. D. Lester, F. A. Ponce, M. G. Craford, D.A. Steigerwald, Appl. Phys. Lett. 66, (1995) 1249. 
[4] W. Qian, M. Skowronski, M. DeGraef, K. Doverspike, L. B. Rowland, D.K. Gaskill, Appl. Phys. Lett. 66, (1995) 1252.

[5] X. H. Wu, L.M. Brown, D. Kapolnek, S. Keller, B. Keller, S.P. DenBaars, J. S. Speck, J. Appl. Phys. 80, (1996) 3228.

[6] B. Garni, J. Ma, N. Perkins, J. Liu, T. F. Kuech, M.G. Lagally, Appl.Phys.Lett. 68, (1996) 1380.

[7] S.J.Rosner, E.C. Carr, M.J.Ludowise, G.Girolami, H.I. Erikson, Appl.Phys.Lett. 70, (1997) 420.

[8] S. Keller, B. P. Keller, Y.-F. Wu, B. Heying, D. Kapolnek, J. S. Speck, U. K. Mishra, and S. P. DenBaars, Appl. Phys. Lett. 68, (1996) 1525.

[9] A. Bykhovski, B. Gelmont and M. Shur, J. Appl. Phys. 74 (1993) 6734.

[10]M. Sumiya, M. Tanaka, K. Ohtsuka, S. Fuke, T. Ohnishi, I. Ohkubo, M. Yoshimoto, H. Koinuma, and M. Kawasaki, Appl. Phys. Lett. 75, 674 (1999).

[11] A. Kazimirov, G. Scherb, J. Zegenhagen, T. L. Lee, M. J. Bedzyk, M. K. Kelly, H. Angerer, and O. Ambacher, J. Appl. Phys. 84, 1703 (1998).

[12] A. R. Smith, R. M. Feenstra, D. W. Greve, M.-S. Shin, M. Skowronski, J. Neugebauer, and J. E. Northrup, Appl. Phys. Lett. 72, 2114 (1998); also see Phys. Rev. Lett. 79, 3934 (1997).

[13] O. H. Hughes, D. Korakakis, T. S. Cheng, A. V. Blant, N. J. Jeffs, and C. T. Foxon, J. Vac. Sci. Technol. B16, 2237 (1998).

[14] B. Daudin, J. L. Rouviere, and M. Arlery, Appl. Phys. Lett. 69, (1996) 2480.

[15] U. Karrer, O. Ambacher, and M. Stutzmann, Appl. Phys. Lett. 77, 2012 (2000).

[16] P. Visconti, K. M. Jones, M. A. Reshchikov, R. Cingolani, H. Morkoç, and R. J. Molnar, Appl. Phys. Lett. 77, 3532 (2000); also see Appl Phys. Lett. 77, 3743 (2000).

[17] E. S. Hellman, MRS Internet J. Nitride Semicond. Res. 3, 11 (1998).

[18] T.Kozawa, T.Kachi, T.Ohwaki, Y.Taga, N.Koide, M.Koike, J.Electr.Soc., 143 (1), L17 (1996).

[19] S. K. Hong, T. Yao, B. J. Kim, S.Y. Yoon, T. I. Kim, Appl. Phys. Lett., 77, 82 (2000)

[20] K. Shiojima, J. Vac. Sci. Technol. B 18 (1), 37 (2000)

[21] C. Youtsey, L. T. Romano, I. Adesida, Appl. Phys. Lett. 73, (1998) 797.

[22] C. Youtsey, L. T. Romano, R. J. Molnar, I. Adesida, Appl. Phys. Lett. 74, (1999) 3537. 